\title{
Atenção primária à saúde bucal no Brasil: processo de trabalho das equipes de saúde bucal
}

\author{
Primary dental healthcare in Brazil: the work process \\ of oral health teams
}

Matheus Neves (https://orcid.org/0000-0002-7336-8987) ${ }^{1}$

Jessye Melgarejo do Amaral Giordani (https://orcid.org/0000-0002-3825-9734) ${ }^{2}$

Fernando Neves Hugo (https://orcid.org/0000-0003-2222-7719) ${ }^{1}$
${ }^{1}$ Departamento de Odontologia Preventiva e Social, Faculdade de Odontologia, Universidade Federal do Rio Grande do Sul. R. Ramiro Barcelos 2492, Santa Cecília. 90035 004 Porto Alegre RS Brasil. matineves@gmail.com ${ }^{2}$ Curso de Odontologia, Universidade Federal de Santa Maria. Santa Maria RS Brasil.

\begin{abstract}
Ensuring access to dental care services requires the development of healthsurveillance practices to ensure comprehensive health care. The objective of this study was toinvestigate the association between social and economic indicators of Brazilian municipalities, work process characteristics, and performance of a list of curative dental procedures by oral health teams. It involved an exploratory, cross-sectional study withmulticenter data collection from 11,374 oral health teams assessed by the National Program for Improvement of Access to and Quality of Primary Healthcare. Multilevel Poisson regression was used to obtain the prevalence of curative dental procedures, which was $69.51 \%$. The social/economic and work variables that remained associated with the outcome included municipalities in which the proportion of primary care-sensitive admissions was below 28\% and that of tooth extractions below 8\%; and oral health teams classified as type II (including oral health assistant and technician) that had different materials available and better work processes. This multilevel analysis, which took into consideration the performance of curative dental care in Brazil, reveals a worrying oral healthcare scenario.

Key words Health service evaluation, Dental healthcare services, Public health dentistry, Health care quality, access, and evaluation
\end{abstract}

Resumo A garantia de atenção odontológica passa pelo desenvolvimento de práticas pautadas na vigilância em saúde, a fim de concretizar a integralidade. Objetivou-se avaliar a associação entre aspectos contextuais dos municipios brasileiros, características do processo de trabalho $e$ a realização de um rol de procedimentos odontológicos curativos pelas equipes de saúde bucal (ESB). Trata-se de estudo exploratório transversal cuja coleta multicêntrica de dados se deu em 11.374 ESB avaliadas pelo Programa Nacional de Melhoria do Acesso e da Qualidade da Atenção Básica. Foi empregada regressão de Poisson multinível para obtenção da prevalência de realização de procedimentos odontológicos curativos, que foi de 69,51\%. As variáveis contextuais e da equipe de saúde que se mantiveram associadas ao desfecho incluíram municípios cuja proporção de internações sensíveis à atenção básica foi menor que $28 \%$ e cuja proporção de exodontias foi menor que $8 \%$; bem como ESB de modalidade II que tinham à disposição materiais, insumos e melhores processos de trabalho. Esta análise multinivel, que considera o desempenho da atenção odontológica curativa no Brasil, aponta para um cenário de atenção odontológica preocupante.

Palavras-chave Avaliação de serviços de saúde, Serviços de saúde bucal, Odontologia em saúde pública; Qualidade, Acesso e Avaliação da Assistência à Saúde 


\section{Introdução}

Entende-se que a integralidade, como um dos princípios doutrinários do Sistema Único de Saúde (SUS), propõe-se a unir as ações direcionadas à concretização da saúde como direito e como serviço, possibilitando uma dimensão da oferta de ações capaz de responder às necessidades de saúde de uma determinada populaçãoº Assim, cria-se o desafio de construir um sistema público de saúde que, para além da atenção às necessidades de saúde do povo brasileiro, constitua-se integral ao engrenar políticas, programas, ações, práticas e cuidados em saúde.

Nesse horizonte, surge, em 2004, o marco teórico da saúde bucal brasileira, a Política Nacional de Saúde Bucal (PNSB), cujo objetivo está em ampliar e garantir atenção odontológica a toda a população. Para isso, busca-se o desenvolvimento de práticas pautadas na vigilância em saúde por meio de ações de promoção, prevenção, tratamento e reabilitação, organizadas em todos os níveis de atenção, concretizando o princípio da integralidade $^{2}$.

Segundo as diretrizes da PNSB, as ações e os serviços odontológicos devem resultar de um adequado conhecimento da realidade de saúde da população, para, assim, construir uma prática efetivamente resolutiva ${ }^{2}$. Desta forma, os serviços odontológicos curativos instituídos e prestados no âmbito da atenção primária à saúde devem se ocupar das patologias mais prevalentes nas comunidades. Os principais agravos que acometem a saúde bucal e que têm sido objeto de estudos epidemiológicos em virtude de sua prevalência e gravidade são: a cárie dentária, a doença periodontal, o câncer de boca, os traumatismos dentários, a fluorose dentária, o edentulismo e a maloclusão ${ }^{3}$.

Entretanto, após mais de uma década de vigência da PNSB, ainda se observa um fenômeno de universalização excludente e integralidade seletiva das ações e serviços básicos de saúde bucal ofertados à população ${ }^{4,5}$. Logo, a avaliação e a gestão dos serviços de atenção primária devem valorizar os componentes de estrutura e sua relação com a qualidade dos processos de trabalho em saúde, visando alcançar resultados positivos no estado de saúde dos indivíduos e da população ${ }^{6,7}$. Com base neste entendimento, consolidase a questão norteadora deste estudo.

Diante desse contexto, o Programa Nacional de Melhoria do Acesso e da Qualidade da Atenção Básica (PMAQ-AB), além de institucionalizar os processos de avaliação da qualidade nas rotinas de trabalhadores e gestores dos serviços de saúde, busca ampliar o acesso à atenção primária, com garantia de padrões de qualidade comparáveis em nível nacional, regional e local, de forma a permitir maior transparência e efetividade nas ações governamentais direcionadas à atenção primária. Para tanto, o PMAQ-AB situa a avaliação dos serviços de saúde como estratégia permanente para a tomada de decisão e ação central para a melhoria da qualidade das ações de saúde, sendo esta considerada um atributo fundamental a ser alcançado no SUS ${ }^{8-10}$.

Os primeiros resultados publicados a partir da avaliação do PMAQ-AB sobre as equipes de saúde bucal brasileiras apontam que $45,1 \%$ dos usuários têm dificuldades de acesso à consulta odontológica; quando o obtêm, a garantia de continuidade de tratamento é dificultada nas regiões Norte e Nordeste. O acesso e o tempo de espera nas especialidades ainda constituem problemas da rede de atenção à saúde, entraves esses que configuram motivos para o deslocamento dos usuários a outros serviços ${ }^{11}$.

Idealiza-se, assim, o desafio de construir uma atenção primária que, além de atender às necessidades de saúde, garanta a toda a população acesso e atendimento integral a serviços de saúde de qualidade, haja visto seu primordial papel de coordenar o cuidado em saúde ${ }^{12}$. A sustentabilidade dos sistemas de saúde, baseada no modelo tradicional de atenção, apresenta evidente sinal de esgotamento, com questionamento sobre a cultura da busca pelo especialista e da oferta em regime de livre demanda ${ }^{13}$. Desta forma, faz-se necessário o desenvolvimento de estudos que efetivamente demonstrem os resultados e o impacto das ações odontológicas realizadas pela equipe de saúde bucal na atenção primária à saúde ${ }^{13}$.

Em vista disso, este estudo teve como objetivo avaliar a associação entre aspectos contextuais sociodemográficos, indicadores do sistema de saúde dos municípios brasileiros e características do processo de trabalho e a realização de um rol de procedimentos odontológicos curativos pelas equipes de saúde bucal avaliadas durante o primeiro ciclo do PMAQ-AB no Brasil.

\section{Métodos}

Trata-se de um estudo transversal, de natureza exploratória, cuja coleta de dados foi realizada de forma multicêntrica, envolvendo 17.479 equipes de atenção básica de todo o Brasil, avaliadas durante o primeiro ciclo do PMAQ-AB. Para este estudo, foram consideradas 11.374 equipes de 
saúde bucal da atenção primária à saúde, correspondendo a $91,7 \%$ do total das 12.403 equipes de saúde bucal avaliadas no país, incluídas conforme esquema apresentado na Figura 1.

Como fonte de dados, este estudo utilizou a avaliação externa do primeiro ciclo do PMA$\mathrm{Q}-\mathrm{AB}$, informações do Instituto Brasileiro de Geografia e Estatística (IBGE) ${ }^{14}$ e o Índice de Desempenho do Sistema Único de Saúde (IDSUS), representado por um conjunto de indicadores simples e compostos que buscam fazer uma aferição contextualizada do desempenho do Sistema de Único de Saúde (SUS) com base em seus princípios e diretrizes ${ }^{15}$.

Para melhor entendimento do PMAQ-AB, deve-se observar sua nota metodológica, segundo a qual o programa é composto por: 1) adesão e contratualização do município ao programa; 2) desenvolvimento de ações de qualificação dos processos de trabalho e de gestão; 3) avaliação externa, para verificar os resultados das equipes e dos gestores da atenção básica; e 4) recontratualização, a fim de incrementar os indicadores de qualidade e estimular a sistematização da avaliação pelo PMAQ-AB ${ }^{16}$. Esse ciclo contínuo de avaliação visa à indução de processos que assegurem maior acesso e qualidade na atenção básica ${ }^{16}$.

$\mathrm{O}$ instrumento de avaliação externa do primeiro ciclo do PMAQ-AB foi organizado em três módulos: I - Observação nas unidades básicas de saúde; II - Entrevista com a equipe de atenção básica e verificação de documentos; e III - Entrevista com os usuários dos serviços de atenção básica sobre satisfação e condições de acesso e utilização dos serviços de saúde. Os dados do presente estudo referem-se às avaliações do módulo I, em que as condições de infraestrutura foram verificadas em um censo das unidades básicas de saúde do Brasil, e do módulo II, respondido pelos profissionais de saúde, mediante entrevista e verificação de documentações in loco.

O período de coleta de dados, pela aplicação dos instrumentos avaliativos, ocorreu entre maio e dezembro de 2012. Para o trabalho em campo, avaliadores externos foram selecionados e treinados uniformemente por meio da utilização de um manual de campo elaborado pelo Departamento de Atenção Básica do Ministério da Saúde e por instituições de ensino e pesquisa parceiras do PMAQ-AB. Cabe ressaltar que os instrumentos avaliativos foram produzidos por meio de um consenso entre pares e previamente testados ${ }^{16}$.

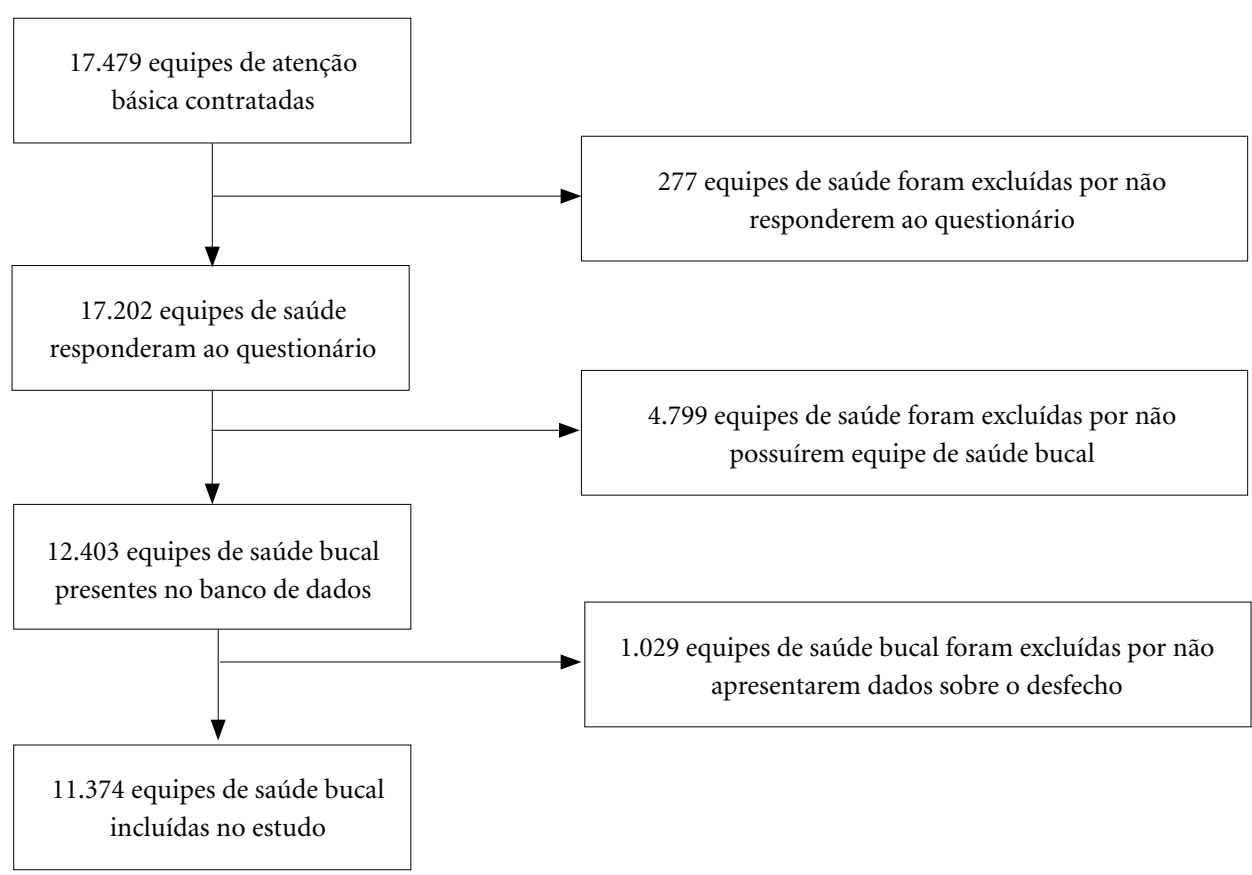

Figura 1. Fluxograma demonstrando a inclusão e exclusão das equipes de saúde bucal no estudo. 
Os avaliadores utilizaram computadores portáteis do tipo tablet para a compilação das informações aferidas. Após a realização da avaliação externa, os dados coletados foram enviados via internet a um servidor do Ministério da Saúde para que fossem validados e então passassem a compor o banco de dados do PMAQ-AB.

O desfecho deste estudo foi a realização de procedimentos odontológicos curativos, variável derivada da realização dos seguintes procedimentos odontológicos: restaurações de amálgama e resina; exodontias; raspagem, alisamento e polimento periodontal; curativo de demora; e drenagem de abscesso dentoalveolar. Tais variáveis foram observadas junto ao módulo II, e o desfecho foi dicotomizado entre as equipes de saúde bucal que realizavam ou não a totalidade daquele rol de procedimentos odontológicos curativos.

As variáveis independentes foram estudadas em duas disposições: variáveis contextuais e variáveis relacionadas às equipes de saúde bucal. As exposições do nível contextual abordaram aspectos sociodemográficos e do sistema de saúde dos municípios avaliados pelo PMAQ-AB. Os aspectos sociodemográficos incluíram as seguintes variáveis: macrorregião nacional, porte populacional do município e índice de desenvolvimento humano (IDH). Para compor as variáveis referentes ao sistema de saúde, utilizaram-se os seguintes indicadores: cobertura de saúde bucal, proporção de internações sensíveis à atenção básica e proporção de exodontias entre procedimentos odontológicos selecionados.

Para a descrição das macrorregiões brasileiras, utilizou-se a classificação do IBGE: Nordeste, Norte, Centro-Oeste, Sul e Sudeste ${ }^{14}$. Para classificar o porte populacional dos municípios, essa variável foi categorizada em menor ou igual a 5 mil habitantes, entre 5 e 10 mil habitantes, entre 10 e 50 mil habitantes, entre 50 e 100 mil habitantes, entre 100 e 500 mil habitantes e mais de 500 mil habitantes. O IDH foi classificado em baixo $(\leq 0,62)$, médio (entre 0,6201 e 0,70$)$ e alto $(>0,70)^{17}$. O indicador de cobertura de saúde bucal foi categorizado em municípios com até 4 mil habitantes adscritos a uma equipe de saúde bucal e municípios cuja proporção foi maior ${ }^{15}$. Para os indicadores relacionados ao sistema de saúde, utilizaram-se os parâmetros elencados no IDSUS $^{15}$, a saber: $28,6 \%$ de internações sensíveis à atenção básica em relação a todas as internações clínicas ${ }^{18}$; e $8 \%$ de exodontias em relação ao total de procedimentos odontológicos básicos realizados em residentes de determinado município em um ano. Tais indicadores foram catego- rizados em acima ou abaixo dos parâmetros de referência.

Para compor as exposições do nível relacionado às equipes de saúde bucal, incluíram-se a presença de equipamentos e insumos para a atenção odontológica (alta e baixa rotação, fotopolimerizador, amálgama, fios de sutura, anestésicos e resinas fotopolimerizáveis), o acolhimento, a resposta ao acolhimento segundo avaliação de risco e vulnerabilidade, a continuidade do cuidado, a realização de visita domiciliar pelo cirurgião-dentista e a modalidade da equipe de saúde bucal.

No que se refere às variáveis correspondentes à disponibilidade de equipamentos e insumos para atenção odontológica, todos os itens avaliados foram categorizados em disponíveis ou indisponíveis. O acolhimento foi definido pela questão "Existe acolhimento à demanda espontânea específica para saúde bucal?", com respostas sim ou não. A resposta ao acolhimento foi investigada pela questão "Oferta é definida em função do risco identificado?" (sim ou não). A presença ou não de continuidade do cuidado foi avaliada com a pergunta "A equipe garante agenda para a continuidade do tratamento de um usuário que iniciou seu tratamento?" (sim ou não). A realização da visita domiciliar foi identificada através da questão "Quais profissionais da equipe realizam cuidado domiciliar?", sendo que o cirurgiãodentista deveria ser apontado como um daqueles profissionais. Por fim, a modalidade da equipe de saúde bucal foi avaliada pela variável "quantidade de profissionais da equipe mínima de atenção básica", em que cirurgião-dentista, técnico em saúde bucal e auxiliar em saúde bucal deveriam ser identificados; esta variável foi categorizada em "não há auxiliar em saúde bucal nem técnico em saúde bucal", "Modalidade I - há auxiliar em saúde bucal ou técnico em saúde bucal" e "Modalidade II - há auxiliar em saúde bucal e técnico em saúde bucal", conforme legislação vigente ${ }^{19}$.

As variáveis independentes que compõem o nível referente às características da equipe de saúde e aos insumos disponíveis à atenção odontológica foram identificadas nos módulos I e II do instrumento de avaliação externa do PMAQ $-\mathrm{AB}^{20}$.

Utilizou-se a abordagem hierárquica para avaliar a associação entre as variáveis contextuais sociodemográficas e do sistema de saúde e as características das equipes de saúde bucal e equipamentos e insumos disponíveis para atenção odontológica, seguindo a metodologia de análise hierárquica proposta por Fuchs et al. ${ }^{21}$, conforme 
demonstra a Figura 2. As análises foram realizadas no software Stata versão 11.

Inicialmente, descreveram-se as variáveis contextuais e referentes à equipe de saúde bucal por meio de frequências absolutas e relativas. A seguir, foi empregada análise multinível para avaliar a associação entre as exposições e o desfecho, com controle de confusão para as variáveis independentes em cada nível de análise ${ }^{21-23}$ Por fim, foi utilizado um modelo de regressão de Poisson multinível (comando xtpoisson, com o subcomando re para os efeitos randômicos) para a obtenção das razões de prevalência (RP) brutas e ajustadas, com seus respectivos intervalos de confiança de 95\% (IC95\%) e nível de significância de $5 \%$.

Após as análises brutas realizadas em cada nível (contextual e equipes de saúde), passou-se à análise ajustada, em que as variáveis do nível contextual com $\mathrm{p}<0,10$ foram consideradas fatores de ajuste ou controle de confusão para as variáveis relativas às equipes de saúde. As variáveis que apresentaram $\mathrm{p}<0,05$ foram consideradas associadas ao desfecho.
A realização deste estudo obteve aprovação prévia do Comitê de Ética em Pesquisa da Universidade Federal do Rio Grande do Sul.

\section{Resultados}

A prevalência de realização de procedimentos odontológicos curativos (restaurações de amálgama e resina, exodontias, raspagem, alisamento e polimento periodontal, curativo de demora e drenagem de abscesso dentoalveolar) foi de 69,51\% (7.906/11.374; IC95\% 68,66-70,35).

Em relação à prevalência de realização de procedimentos odontológicos curativos, as regiões Sudeste $(79,8 \%)$ e Sul $(75,4 \%)$ apresentaram maiores prevalências quando comparadas às demais. A maior ocorrência do desfecho foi nas cidades com mais de 500 mil habitantes $(78,1 \%)$, nas cidades com IDH superior a $0,7(76,6 \%)$ e com cobertura de saúde bucal de até 4 mil habitantes $(70,4 \%)$. Além disso, houve maior prevalência do desfecho quando a proporção de internações sensíveis à atenção básica foi menor que
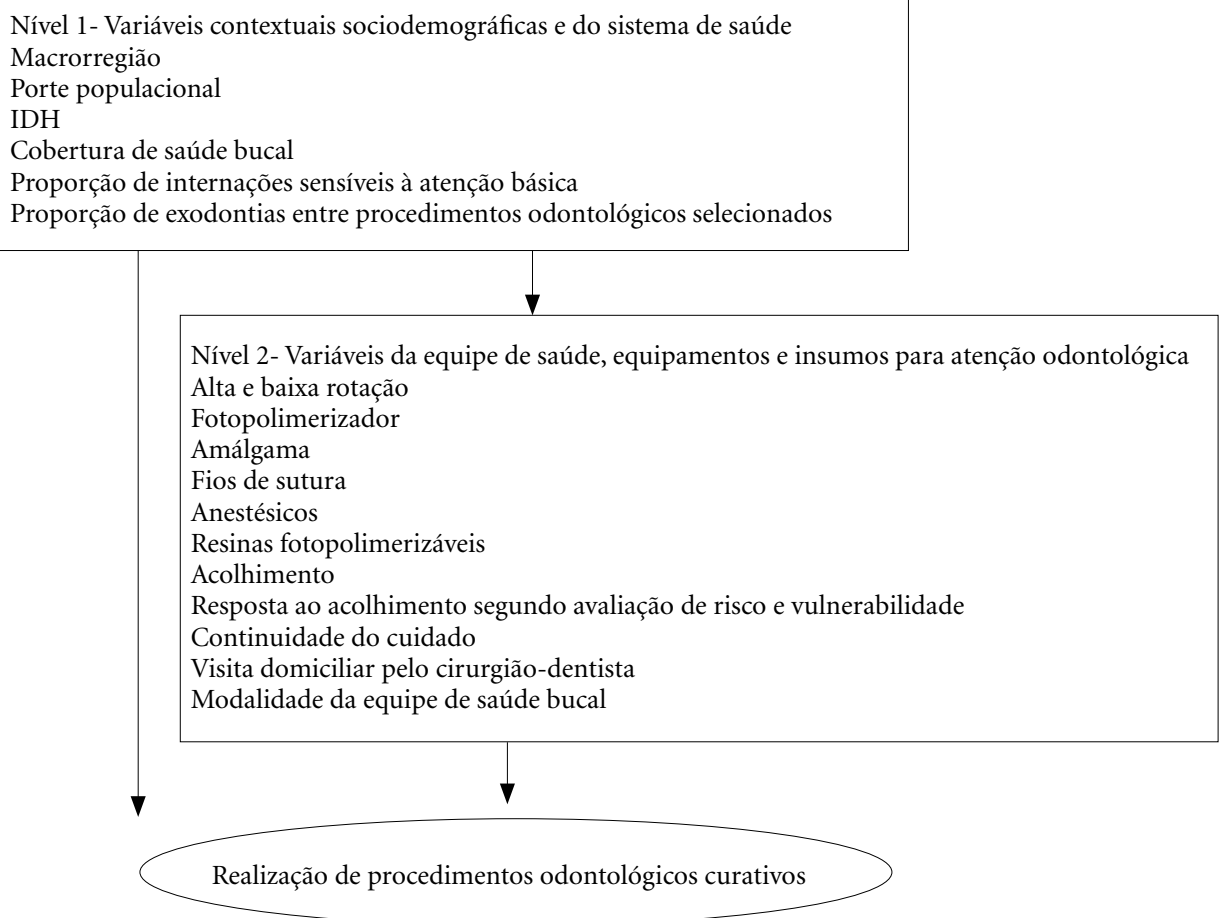

Figura 2. Modelo teórico hierárquico utilizado para avaliar a associação das variáveis contextuais e características das equipes de saúde bucal com a realização de procedimentos curativos. IDH = índice de desenvolvimento humano. 
$28 \%(77,4 \%)$ e quando a proporção de exodontias entre procedimentos selecionados foi menor que $8 \%(75,5 \%)$ (Tabela 1$)$.

$\mathrm{Na}$ análise ajustada para variáveis contextuais sociodemográficas e do sistema de saúde, a prevalência do desfecho na região Sudeste foi $62 \%$ maior do que na região Norte (RP 1,62; IC95\% $1,44-1,83)$. Entre as variáveis relacionadas ao sistema de saúde, a probabilidade de ocorrência de procedimentos odontológicos curativos foi $8 \%$ e $6 \%$ maior, respectivamente, nos municípios com proporção de internações sensíveis à atenção básica $<28 \%$ e quando a proporção de exodontias entre os procedimentos selecionados foi $<8 \%$ (Tabela 1).

Em relação às variáveis elencadas no nível da equipe de saúde e dos equipamentos e insumos para atenção odontológica, observou-se maior prevalência do desfecho quando havia alta e baixa rotação $(70,0 \%)$, presença de amálgama (70,3\%), ausência de anestésico $(53,5 \%)$, presença de resinas fotopolimerizáveis $(70,9 \%)$, fios de sutura $(70,6 \%)$ e fotopolimerizador $(70,9 \%)$. Ainda, as variáveis presença de acolhimento, resposta ao acolhimento segundo avaliação de risco e vulnerabilidade, continuidade do cuidado, visita domiciliar pelo cirurgião-dentista e presença de equipe de saúde bucal na modalidade II foram associadas a maior ocorrência de procedimentos odontológicos curativos (Tabela 2).

$\mathrm{Na}$ análise hierárquica, após os ajustes, entre os fatores do nível equipe de saúde, a presença de alta e baixa rotação e a disponibilidade de anestésicos mostraram menor probabilidade de associação com o desfecho (RP 0,73, IC95\% 0,61-0,87; e RP 0,68, IC95\% 0,57-0,83, respectivamente; $\mathrm{p}$ $<0,001)$. Presença de acolhimento aumentou em $24 \%$ a prevalência de procedimentos odontológicos curativos (RP 1,24; IC95\% 1,15-1,34); presença do cirurgião-dentista na visita domiciliar aumentou a probabilidade do desfecho em $8 \%$ (p $<0,001)$; o mesmo foi observado quando havia a continuidade do cuidado (prevalência do desfecho $26 \%$ maior). A modalidade da equipe de saúde bucal também influenciou o desfecho: quando a modalidade II estava presente, a ocorrência foi 1,15 vez maior do que quando não havia auxiliar em saúde bucal nem técnico em saúde bucal (RP 1,15; IC95\% 1,00-1,31) (Tabela 2).

A variância (deviance) no modelo vazio (sem as variáveis independentes) foi de -10.781,16, e este valor reduziu para $-10.172,16$ na análise multivariada.

\section{Discussão}

Neste estudo, 11.374 equipes de saúde bucal do Brasil foram avaliadas quanto à realização de um rol de procedimentos odontológicos de caráter curativo, no intuito de explorar sua associação com aspectos contextuais sociodemográficos, indicadores do sistema de saúde dos municípios brasileiros e características do processo de trabalho das equipes de saúde bucal avaliadas durante o primeiro ciclo do PMAQ-AB.

Evidenciou-se que, entre as equipes de saúde bucal avaliadas em todo o território nacional, $30,49 \%(3.468 / 11.374)$ não realizam um ou mais dos procedimentos odontológicos compilados como desfecho, quais sejam: restaurações de amálgama e resina, exodontias, raspagem, alisamento e polimento periodontal, curativo de demora e drenagem de abscesso dentoalveolar.

Os achados deste estudo enfatizam importantes diferenças entre as macrorregiões nacionais, sendo que as regiões Sul e Sudeste apresentaram as maiores prevalências de procedimentos odontológicos curativos; em contrapartida, as regiões Norte e Nordeste apresentaram as menores prevalências. $\mathrm{O}$ gradiente social tem sido evidenciado como modulador da saúde bucal em várias populações de diferentes países, independentemente da natureza, da abrangência e da eficiência dos respectivos sistemas de saú$\mathrm{de}^{24}$. Estudos realizados tanto no nível individual quanto no ecológico comprovam a relação entre desigualdades sociais e condição de saúde bucal, sugerindo a existência de iniquidades ${ }^{25}$.

O Brasil é um país marcado por desigualdades socioeconômicas, com potencial impacto na saúde $^{26}$. As políticas de saúde do país têm sido orientadas no sentido de tentar minimizar tais iniquidades. Por meio de uma breve recuperação documental, tem-se que a Norma Operacional Básica de 1996 (NOB/SUS 1996) ${ }^{27}$, do Ministério da Saúde, orienta o SUS em direção à equidade. Desde então, vários estudos têm observado uma tendência redistributiva da política de transferência de recursos financeiros do SUS por meio do piso de atenção básica, que tem favorecido municípios com baixo IDH ${ }^{25}$.

Em 2006, mais um passo em direção à equidade foi dado, com o Pacto pela Saúde ${ }^{28}$. Mais além, em 2011, agregou-se o Contrato Organizativo de Ação pela Saúde (COAP) ${ }^{29}$, que objetiva a organização e a integração de ações e serviços em regiões de saúde, a fim de garantir integralidade de atenção aos usuários. Por fim, a Política Nacional da Atenção Básica publicada em 2006, e 


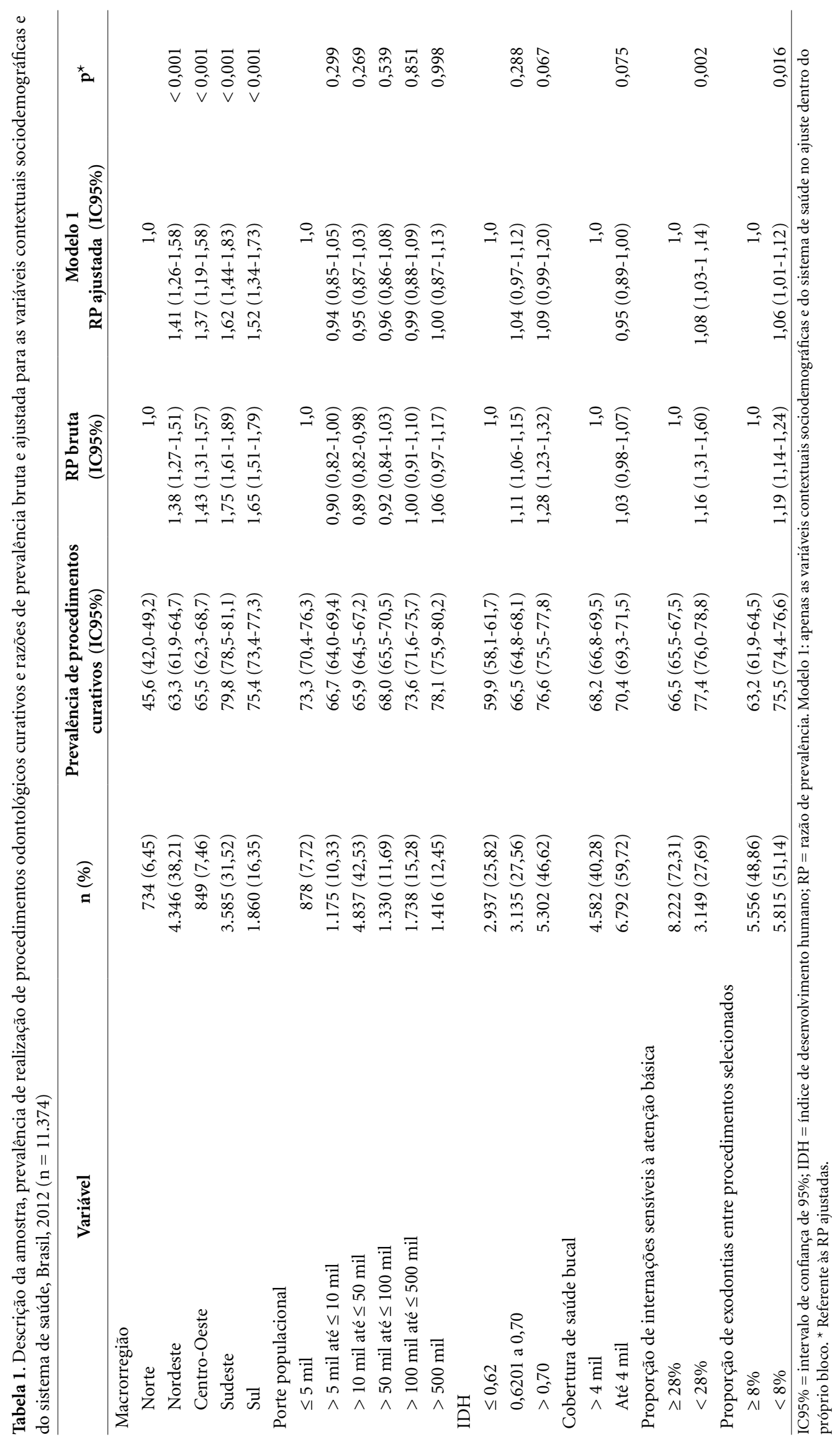




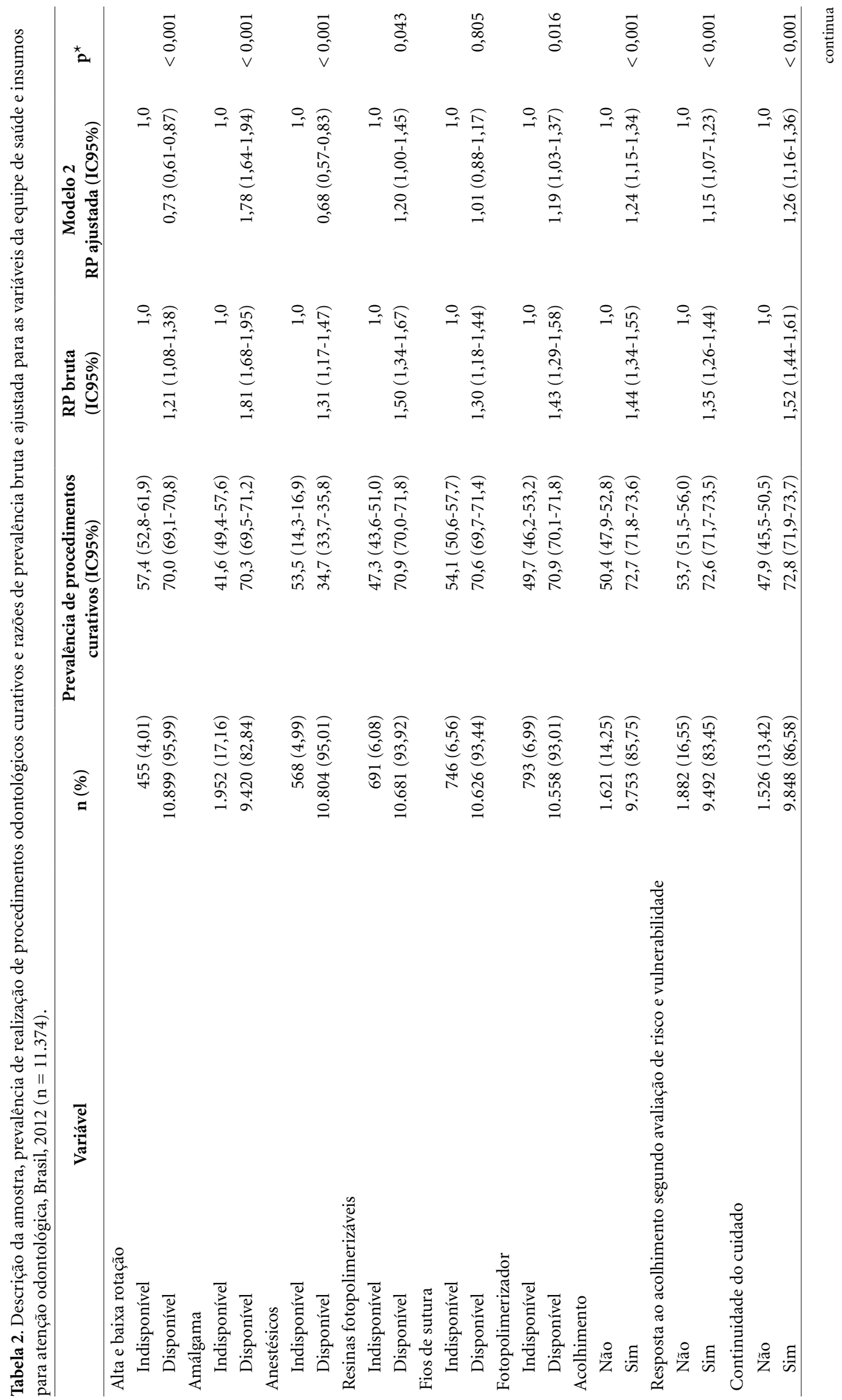


revisada em 2011 (PNAB) $^{30}$, incorpora o PMAQ $-\mathrm{AB}^{20}$ como uma estratégia de indução de melhorias no acesso e na qualidade da atenção básica, ratificando a saúde bucal na agenda de prioridades de gestão.

Neste estudo, maior prevalência do desfecho também ocorreu quando a proporção de internações sensíveis à atenção básica foi menor que $28 \%$ e quando a proporção de exodontias entre procedimentos selecionados foi menor que $8 \%$. As evidências científicas convergem ao apresentar os efeitos da implantação da Estratégia Saúde da Família na saúde da população, associando-a à redução da mortalidade infantil, ao declínio de hospitalizações evitáveis, ao aumento da oferta de trabalho entre os adultos e ao aumento da matrícula escolar ${ }^{31}$.

Entretanto, a evidência disponível em relação à saúde bucal tem se caracterizado, sobretudo, por pesquisas de avaliação de processos, cujos desfechos são indicadores de uso e de acesso dos serviços de saúde ${ }^{25,31}$. Os indicadores de saúde bucal constituem uma importante ferramenta para a avalição dos recursos destinados a essa área na atenção básica e também para a avaliação do impacto da aplicação desses recursos na população. Portanto, esses indicadores devem ser considerados na implementação de políticas públicas de forma equânime ${ }^{25}$.

Em recente estudo, Ely et al. ${ }^{31}$ avaliaram o resultado da atuação das equipes de saúde bucal no sul do Brasil e observaram que jovens de áreas não cobertas por equipes de saúde bucal tiveram quase a metade da perda de dentes dos adolescentes das áreas cobertas por equipes de saúde bucal, sugerindo que a expansão do acesso com a inserção de equipes de saúde bucal não se traduziu em melhor saúde bucal. Os autores enfatizam a importância da determinação social na saúde bucal e destacam a necessária revisão dos processos de trabalho assumidos pela equipe de saúde bucal, achados também constantes no presente estudo.

As exposições avaliadas no nível da equipe de saúde evidenciam a importância da disponibilidade de equipamentos e insumos para a atenção odontológica, enfatizando que atenção odontológica de qualidade requer a presença de equipamentos, instrumentais e insumos mínimos e adequados. Um recente estudo sueco concluiu que a estrutura física do ambiente de trabalho influencia diretamente a produtividade e a eficiência dos dentistas ${ }^{32}$. Já no Brasil, a evidência científica aponta para a necessidade de melhorias nas condições de trabalho, bem como na infraestrutura para o trabalho do dentista no SUS $^{33}$. 
Uma análise descritiva do PMAQ-AB de Pernambuco observou que a maioria das equipes de saúde bucal refere a disponibilidade dos equipamentos necessários para a realização das ações e serviços de saúde bucal. Em pouco menos de $20 \%$ das equipes, a disponibilidade de insumos odontológicos para procedimentos restauradores foi limitada. Quanto aos procedimentos odontológicos clínico-cirúrgicos, os achados demonstram sua realização rotineira ${ }^{34}$. Já na Paraíba, as unidades de saúde apresentam alguns equipamentos e insumos em número reduzido, com diferença significativa entre o tipo de unidade e as regionais de saúde do estado ${ }^{35}$.

Ainda com relação às variáveis aferidas no nível da equipe de saúde, a realização de acolhimento, avaliação de risco e vulnerabilidade, oferta de cuidado continuado e visita domiciliar realizada pelo cirurgião-dentista que integra uma equipe em modalidade II, com a presença de auxiliar e técnico em saúde bucal, apresentaram associação estatisticamente significante com a realização de um rol de procedimentos curativos.

Tais achados reforçam que as mudanças no sistema de trabalho em saúde bucal estão intimamente associadas ao modo como são produzidas as ações de saúde e à maneira como os serviços de saúde e o Estado se organizam para produzi-las e, mais além, distribuí-las ${ }^{36}$. O processo de trabalho em saúde bucal não se esgota na assistência odontológica individual; a integralidade só é alcançada por meio da criação de redes macro e microinstitucionais, em processos dinâmicos, voltadas ao fluxo de assistência ao usuário e centrada em seu campo de necessidades. Achados iniciais a partir do PMAQ-AB revelam que a constituição das linhas de cuidado é um dos pontos mais significativos na busca pela integralidade e se dá pela organização dos processos de trabalho na atenção básica, com assistência multiprofissional, operando com acolhimento, vinculação de clientela e responsabilidade da equipe ${ }^{34}$.

Aqui, a reflexão recai sobre a equipe de saúde, que precisa romper com o sistema hegemônico de atenção à saúde centrada na assistência curativa, hospitalar e superespecializada, na vertente de interesses econômicos e corporativos. Deve, em vez disso, atuar conforme um sistema universal, que busca modelos de atenção que valorizem a integralidade, o cuidado humanizado e a promoção da saúde. Gonzáles e Almeida ${ }^{37}$ defendem que tal mudança depende do perfil de formação e da prática dos profissionais de saúde. Rocha et al..$^{38}$ indicam que o investimento na formação de profissionais e na sua fixação pode contribuir significa- tivamente para melhorar os índices de eficiência obtidos pelas equipes de saúde da atenção básica.

Nesse sentido, dados descritivos do PMAQ $-\mathrm{AB}$ apontam, quanto ao processo de trabalho, que houve compartilhamento de agenda entre o cirurgião-dentista e os demais profissionais que compõem as equipes de atenção básica em $78,5 \%$ das equipes avaliadas. Entretanto, em apenas pouco mais de $40 \%$ das equipes, o cirurgiãodentista participa do acolhimento à demanda espontânea ${ }^{34}$.

Mesmo que as evidências científicas já tenham apontado a flagrante necessidade de evolução do modelo assistencial e dos processos de trabalho, há que se refletir se mesmo após mais de uma década de políticas de ampliação de acesso e cobertura de saúde bucal a odontologia brasileira segue tecnicamente elogiável, cientificamente discutível e socialmente caótica ${ }^{39}$. Segundo Ceccim e Feuerwerker ${ }^{40}$, uma política ativa de mudança na formação irá possibilitar uma melhor articulação entre universidade e serviços de saúde. Com o processo de aproximação e construção de compromissos, a responsabilidade pública e a relevância social da universidade serão ampliadas na medida em que trazem para o contexto da educação o conjunto das diretrizes do SUS, em especial a integralidade.

Defender a integralidade, por fim, não implica deixar de lado todos os conhecimentos técnicos sobre as doenças. A integralidade não existe sem conhecimento técnico. O que se busca é um uso prudente desse conhecimento sobre doença, mas, acima de tudo, um uso guiado por uma visão abrangente das necessidades dos sujeitos que tratamos. Portanto, fica claro que a formação dos profissionais não pode ser apenas técnica: precisa compreender cultura, sistema e relação ${ }^{37}$.

\section{Considerações finais}

Os resultados apresentados exploram e caracterizam aspectos contextuais sociodemográficos, indicadores do sistema de saúde dos municípios brasileiros, equipamentos, insumos e características do processo de trabalho, sob a ótica conceitual da integralidade. Entretanto, a leitura das informações precisa considerar, nesse caso, tanto o limite explicativo dos dados exclusivamente quantitativos, em face da complexidade e variabilidade das modelagens assistenciais que operam simultaneamente no país, como a natureza da coleta, que tem nos resultados da avaliação externa desdobramentos políticos e financeiros para 
a gestão municipal. Deve-se considerar também que os dados se referem somente às equipes com saúde bucal que aderiram ao PMAQ-AB, e não ao universo das unidades básicas de saúde do país.

Reafirma-se que a PNSB e seus mais de 10 anos de implantação trouxeram a necessária e oportuna ampliação do número de equipes de saúde bucal no país; porém, esta análise multinível que considera o desempenho da atenção odontológica curativa junto ao universo de equipes participantes do ciclo I do PMAQ-AB revela achados que estão longe de ser considerados satisfatórios. Está identificada a necessidade de mudança no modelo assistencial em saúde bucal. Buscar novos territórios conceituais e explorar práticas inovadoras são elementos indispensáveis, mas não suficientes para superar o paradigma hegemônico de atenção odontológica na atenção básica brasileira.

Por fim, reafirma-se que investimentos na qualificação da gestão ou gerência setorial são condição necessária para a melhoria do acesso e da qualidade da atenção em saúde bucal no país. Além disso, com base no contexto atual, com grande alocação de recursos nos estados e municípios, é crescente a necessidade de práticas de saúde baseadas em evidência, de investigações que permitam melhor quantificar e qualificar o impacto das intervenções e das políticas em saúde pública adotadas no Brasil.

\section{Colaboradores}

M Neves participou da concepção do estudo, organização, análise e descrição dos dados, e produção do artigo científico. JMA Giordani participou da análise e descrição dos dados e contribuiu com a produção do artigo científico. FN Hugo participou da concepção do estudo, organização dos dados, além da produção e revisão final do artigo científico.

\section{Referências}

1. Pucca Junior GA. Política Nacional de Saúde Bucal do Brasil, integralidade e acesso: o caso Brasil Sorridente [dissertação]. Brasília: Universidade de Brasília; 2013.

2. Brasil. Ministério da Saúde (MS). Diretrizes da Política Nacional de Saúde Bucal. Brasília: MS; 2004. [acesso 2016 Abr 10]. Disponível em: http://bvsms.saude.gov. br/bvs/publicacoes/politica_nacional_brasil_sorridente.pdf

3. Brasil. Ministério da Saúde (MS). Saúde bucal. Brasília: MS; 2006. [Cadernos de Atenção Básica, no 17].

4. Bonfada D, Cavalcante JRLP, Araújo DP, Guimarães J. A integralidade da atenção à saúde como eixo da organização tecnológica nos serviços. Cien Saude Colet 2012; 17(2):555-560.

5. Mello AL, Andrade SR, Moyses SJ, Erdmann AL. Saúde bucal na rede de atenção e processo de regionalização. Cien Saude Colet 2014; 19(1):205-214.

6. Silveira DS, Santos IS, Costa JSD. Atenção pré-natal na rede básica: uma avaliação da estrutura e do processo. Cad Saude Publica 2001; 17(1):131-139.

7. Moura BLA, Cunha RC, Fonseca ACF, Aquino R, Medina MG, Vilasbôas ALQ, Xavier AL, Costa AF. Atenção primária à saúde: estrutura das unidades como componente da atenção à saúde. Rev Bras Saude Mater Infant 2010; 10(1):69-81.

8. Pinto HA, Souza ANA, Ferla AA. O Programa Nacional de Melhoria do Acesso e da Qualidade da Atenção Básica: várias faces de uma política inovadora. Saúde Debate 2014; 38(Especial):358-372.

9. Rodrigues V, Santos CRI, Pereira MU. A experiência de planejar e operacionalizar o PMAQ-AB no estado do Acre. Saúde Debate 2014; 38(Especial):173-181.

10. Seidl HMF, Vieira SDP, Fausto MCR, Lima RDCD, Gagno JL. Gestão do trabalho na atenção básica em saúde: uma análise a partir da perspectiva das equipes participantes do PMAQ-2012. Saúde Debate 2014; 38(Especial):94-108. 
11. Casotti E, Contarato PC, Fonseca ABM, Borges PKDO, Baldani MH. Atenção em saúde bucal no Brasil: uma análise a partir da avaliação externa do PMAQ-AB. Saúde Debate 2014; 38(Especial):140-157.

12. Cruz DB, Nascimento AC, Moysés SJ, Ditterich RG, Gabardo MCL. Processo de trabalho na estratégia de saúde da família: uma perspectiva a partir da equipe de saúde bucal. Rev APS 2009; 12(2):168-175.

13. Bulgareli JV, Faria ET, Ambrosano GMB, Vazquez FL, Cortellazzi KL, Meneghim MC, Mialhe FL, Pereira AC. Informações da atenção secundária em odontologia para avaliação dos modelos de atenção à saúde. Rev Odontol UNESP 2013; 42(4):229-236.

14. Instituto Brasileiro de Geografia e Estatística (IBGE). Cidades@ [acessado 2016 Abr 14]. Disponível em: http://www.cidades.ibge.gov.br/xtras/home.php

15. Brasil. Ministério da Saúde (MS). Índice de Desempenho do Sistema Único de Saúde (IDSUS). Fichas detalhadas dos indicadores. [acessado 2016 Abr 14]. Disponível em: http://idsus.saude.gov.br/detalhadas.html

16. Brasil. Ministério da Saúde (MS). Nota Metodológica da certificação das equipes de atenção básica participantes do Programa de Melhoria do Acesso e da Qualidade na Atenção Básica. Brasília: MS; 2013. [acessado 2016 Mar 20]. Disponível em: http://189.28.128.100/dab/docs/ portaldab/documentos/nota_metodologica_pmaq.pdf

17. Programa das Nações Unidas para o Desenvolvimento (PNUD). Atlas do desenvolvimento humano dos municípios. PNUD; 2013. [acessado 2016 Mar 20]. Disponível em: http://www.atlasbrasil.org.br/2013/

18. Brasil. Ministério da Saúde (MS). Portaria no 221, de 17 de abril de 2008. Publica a lista brasileira de internações por condições sensíveis à atenção primária. Diário Oficial da União 2008; 18 abr. Seção 1, p. 70.

19. Brasil. Ministério da Saúde (MS). Portaria no 3.012, de 26 de dezembro de 2012. Redefine a composição das equipes de saúde bucal da Estratégia Saúde da Família constante na Política Nacional de Atenção Básica (PNAB). Diário Oficial da União 2012; 27 dez.

20. Brasil. Ministério da Saúde (MS). Secretaria de Atenção à Saúde, Departamento de Atenção Básica. Programa Nacional de Melhoria do Acesso e da Qualidade da Atenção Básica (PMAQ-AB): manual instrutivo. Brasília: MS; 2012.

21. Fuchs SC, Victora CG, Fachel J. Modelo hierarquizado: uma proposta de modelagem aplicada à investigação de fatores de risco para diarréia grave. Rev Saude Publica 1996; 30(2):168-178.

22. Bastos LS, Oliveira RVC, Velasque LS. Obtaining adjusted prevalence ratios from logistic regression models in cross-sectional studies. Cad Saude Publica 2015; 31(3):487-495.

23. Rabe-Hesketh S, Skrondal A. Multilevel and longitudinal modeling using Stata. $2^{\text {nd }}$ ed. College Station: Stata Press; 2008.

24. Sheiham A, Alexander D, Cohen L, Marinho V, Moysés S, Petersen P, Spencer J, Watt RG, Weyant R. Global oral health inequalities: task group-implementation and delivery of oral health strategies. Adv Dent Res 2011; 23(2):259-267.

25. Fernandes JKB, Pinho JRO, Queiroz RCS, Thomaz EBAF. Evaluation of oral health indicators in Brazil: a trend towards equity in dental care? Cad Saude Publica 2016; 32(2):1-18
26. Paim J, Travassos C, Almeida C, Bahia L, Macinko J. The Brazilian health system: history, advances, and challenges. Lancet 2011; 377(9779):1778-1797.

27. Brasil. Ministério da Saúde (MS). Portaria nº 2.203, de 5 de novembro de 1996. Diário Oficial da União 1996; 6 nov.

28. Brasil. Ministério da Saúde (MS). Pactos pela vida, em defesa do SUS e de gestão. Brasília: MS; 2006. [acessado 2015 Jan 14]. Disponível em: http://www.saude.mppr. mp.br/arquivos/File/volume1.pdf

29. Brasil. Ministério da Saúde (MS). Guia para a elaboração do Contrato Organizativo da Ação Pública: construindo o COAP passo a passo. Brasília: MS; 2014.

30. Brasil. Ministério da Saúde (MS). Portaria no 2.488, de 21 de outubro de 2011. PNAB - Política Nacional de Atenção Básica. Diário Oficial da União 2011; 22 out.

31. Ely HC, Abegg C, Celeste RK, Pattussi MP. Impacto das equipes de saúde bucal da Estratégia da Saúde da Família na saúde bucal de adolescentes do sul do Brasil. Cien Saude Colet 2016; 21(5):1607-1616.

32. Rolander B, Jonker D, Winkel J, Sandsjö L, Balogh I, Svensson E, Ekberg K. Working conditions, health and productivity among dentists in Swedish public dental care-a prospective study during a 5-year period of rationalisation. Ergonomics 2013; 56(9):1376-1386.

33. Moimaz ASS, Rovida TAS, Silva MM, Silva MR, Gardin CAS. Work conditions and occupational health of dentists in Brazilian public health system. Med Segur Trab 2014; 60(234):44-52.

34. Lorena SJE, Martelli PJL, Albuquerque MSV, Lyra TM, Farias SF. Acesso e qualidade: avaliação das equipes de saúde bucal participantes do PMAQ-AB 2012 em Pernambuco. Saúde Debate 2015; 39(104):136-146.

35. Limão NP, Ferreira Filho JCC, Protásio APL, Santiago BM, Gomes LB, Santos ML, Valença AMG. Equipamentos e insumos odontológicos e sua relação com as unidades da atenção primária à saúde. Rev Bras Promoç Saúde 2016; 29(1):84-92.

36. Nickel DA, Lima FG, Silva BB. Modelos assistenciais em saúde bucal no Brasil. Cad Saude Publica 2008; 24(2):241-246.

37. González AD, Almeida MJ. Integralidade da saúde: norteando mudanças na graduação dos novos profissionais. Cien Saude Colet 2010; 15(3):757-762.

38. Rocha TAH, Silva NC, Barbosa ACQ, Rodrigues JM. Human resource management in health and performance of work process in the primary health care-an efficiency analysis in a Brazilian municipality. $J$ Health Manag 2014; 16(3):365-379.

39. Garrafa V, Moysés SJ. Odontologia brasileira: tecnicamente elogiável, cientificamente discutível, socialmente caótica. Divulg Saúde Debate 1996; 13:6-17.

40. Ceccim RB, Feuerwerker LCM. Mudança na graduação das profissões de saúde sob o eixo da integralidade. Cad Saude Publica 2004; 20(5):1400-1410.

Artigo apresentado em 10/04/2017

Aprovado em 26/07/2017

Versão final apresentada em 28/07/2017 